\title{
Neural Underpinnings of Cortisol Effects on Fear Extinction
}

\author{
Christian Josef Merz ${ }^{*,}$, Tanja Christina Hamacher-Dang', Rudolf Stark ${ }^{2,3}$, Oliver Tobias Wolf' and \\ Andrea Hermann ${ }^{2,3}$
}

IInstitute of Cognitive Neuroscience, Department of Cognitive Psychology, Ruhr-University Bochum, Bochum, Germany; ${ }^{2}$ Department of Psychotherapy and Systems Neuroscience, Justus Liebig University Giessen, Giessen, Germany; ${ }^{3}$ Bender Institute of Neuroimaging, Justus Liebig University Giessen, Giessen, Germany

\begin{abstract}
Extinction of conditioned fear embodies a crucial mechanism incorporated in exposure therapy. Clinical studies demonstrated that application of the stress hormone cortisol before exposure sessions facilitates exposure success, but the underlying neural correlates remain unknown. Context- and stimulus-dependent cortisol effects on extinction learning will be characterized in this study and tested in the extinction and in a new context. Forty healthy men participated in a 3-day fear conditioning experiment with fear acquisition in context A (day I), extinction training in context B (day 2), and recall in context B and a new context C one week later (day 3 ). Hydrocortisone (30 mg) or placebo was given before extinction training. Blood-oxygen-level-dependent responses and skin conductance responses (SCRs) served as dependent measures. At the beginning of extinction training, cortisol reduced conditioned SCRs, diminished activation of the amygdala-hippocampal complex, and enhanced functional connectivity of the anterior parahippocampal gyrus with the ventromedial prefrontal cortex (vmPFC). After one week, the cortisol group showed increased hippocampal activation and connectivity to the vmPFC toward an extinguished stimulus and reduced insula activation toward a nonextinguished stimulus in the extinction context. However, this inhibitory cortisol effect did not extend to the new context. Taken together, cortisol reduced fear recall at the beginning of extinction and facilitated the consolidation of the extinction memory as evidenced by an inhibitory activation pattern one week later. The stress hormone exerted a critical impact on the amygdala-hippocampus-vmPFC network underlying fear and extinction memories. However, cortisol did not attenuate the context dependency of extinction.

Neuropsychopharmacology (2018) 43, 384-392; doi:I0.1038/npp.2017.227; published online 25 October 2017
\end{abstract}

\section{INTRODUCTION}

Anxiety as well as trauma- and stressor-related disorders such as posttraumatic stress disorder (PTSD) represent the most common mental disorders (Kessler et al, 2005) leading to tremendous individual and socioeconomic challenges. Exposure therapy incorporated in cognitive behavioral therapy (CBT) constitutes a highly effective treatment approach in these disorders, in particular in phobias, but without being capable of preventing relapses completely (Boschen et al, 2009; Bouton, 2002). One main mechanism of exposure therapy is supposed to rely on the extinction of conditioned fear (Craske et al, 2008; Graham and Milad, 2011). Extinction creates a new inhibitory memory trace competing with the original fear memory trace in a contextdependent way (Bouton, 2004; Vervliet et al, 2013). Thus, the extinction memory trace might be well recalled in the extinction/exposure context but not necessarily in the original acquisition context or completely new contexts. Relapses could therefore develop because of difficulties in

* Correspondence: Dr Cl Merz, Institute of Cognitive Neuroscience, Department of Cognitive Psychology, Ruhr-University Bochum, Universitätsstraße 150, 44780 Bochum, Germany, Tel: +49 234 3224498, Fax: +49 234 14308, E-mail: Christian.J.Merz@rub.de

Received 9 March 2017; revised 2 August 2017; accepted 18 September 2017; accepted article preview online 26 September 2017 extinction memory recall and/or its (long-lasting) transfer beyond the therapy context (Boschen et al, 2009; Vervliet et al, 2013).

Therapy success of exposure therapy might be improved using the stress hormone cortisol that has been extensively shown to modulate emotional learning and memory processes: cortisol inhibits retrieval but enhances consolidation of declarative memories (Schwabe et al, 2012). Indeed, preexposure cortisol application enhanced therapy success in patients with spider, social, or height phobia, also in the long run (de Quervain et al, 2011; Soravia et al, 2006). Corresponding to the literature on declarative memory, it has been suggested that cortisol application reduces fear recall during the encounter of the feared object or situation in exposure therapy and simultaneously facilitates the consolidation of the newly learned information (less phobic fear) enabling an enhanced extinction memory recall at a later point in time (de Quervain and Margraf, 2008; de Quervain et al, 2017). Translating these effects to fear extinction, only one human study so far reported preextinction stress to reduce fear recall (evident in expectancy of the dangerous unconditioned stimulus) at the beginning of extinction and a test session one day later (Bentz et al, 2013).

The crucial brain region involved in these beneficial effects of stress hormones on phobic fear processing seems to be the amygdala (Nakataki et al, 2016) that acts as the central player 
for the acquisition of fear and extinction memories (Quirk and Mueller, 2008). In addition, excitatory input from the (dorsal) anterior cingulate cortex (ACC) and inhibitory input from the ventromedial prefrontal cortex (vmPFC) modulate the expression of fear and extinction memories via the amygdala (Graham and Milad, 2011; Dejean et al, 2015). Importantly, this circuit receives contextual information from the hippocampal complex as shown by converging evidence from rodent and human studies (Maren et al, 2013; Milad et al, 2007; Hermann et al, 2016).

In the present functional magnetic resonance imaging (fMRI) study, we investigated the neural underpinnings of preextinction cortisol administration on the extinction circuit (taking place one day after fear acquisition) and its context dependency. Crucially, the transfer of the assumed cortisol-driven enhanced extinction memory established during extinction learning was tested one week later during recall in the extinction context as well as in a new context. In addition, we explored the modulation of context-dependent fear responding toward a nonextinguished fear stimulus by cortisol. Taken together, this approach provides a valuable model for relapses including context- and stimulusdependent generalization processes.

\section{MATERIALS AND METHODS}

\section{Participants}

In total, 48 healthy male participants were recruited at the local university. Persons reporting MRI exclusion criteria, chronic or acute illnesses, color blindness, regular intake of medicine, current medical or psychological treatment, drug use, age $<18$ or $>35$ years, and body mass index (BMI) $<18$ or $>27 \mathrm{~kg} / \mathrm{m}^{2}$ were not eligible for participation. Righthandedness was assured by the Edinburgh Inventory of Handedness (Oldfield, 1971). All participants had normal or corrected-to-normal vision. They were instructed to refrain from physical exercise and consumption of food and drinks (except water) $2 \mathrm{~h}$ before all testing sessions.

After exclusion of all persons not completing all three experimental days, not acquiring contingency awareness during fear acquisition or showing excessive head motions during scanning, 40 participants remained in the final sample. All participants were reimbursed with $10 € / \mathrm{h}$ for their participation. All procedures were conducted in agreement with the Declaration of Helsinki and approved by the ethics committee of the Faculty of Medicine of the Ruhr-University Bochum (registration number: 4571-13).

\section{Stimulus Material}

Stimuli and procedure were adopted from Milad and colleagues (2007) as implemented before (Hermann et al, 2016). Pictures of an office room, a room with a shelf, and a conference room served as contexts that were assigned as contexts $\mathrm{A}, \mathrm{B}$, or $\mathrm{C}$ by means of permutation. The same desk lamp was included in each of the contexts lighting up in either red, blue, or yellow serving as three conditioned stimuli (CS). The experiment was realized with the Presentation software package (Neurobehavioral Systems, Albany, CA) and presented via fMRI-ready goggles (Visua-Stim Digital; Resonance Technology, Northridge, CA).
A constant voltage stimulator (STM200; BIOPAC Systems, Goleta, CA) provided transcutaneous electrical stimulation serving as the unconditioned stimulus (UCS). Application of the UCS was realized using two electrodes (surface size: $1 \mathrm{~cm}^{2}$ ) attached to the fingertips of the second and third fingers of the right hand. The intensity of the electrical stimulation was set individually to be 'unpleasant but not painful' by use of a gradually increasing rating procedure.

\section{Procedure}

Individual sessions were conducted in the afternoon (between 1230 and $1900 \mathrm{~h}$ ) on 2 consecutive days for acquisition and extinction and one week later for recall. The duration of experimental sessions was $\sim 60$ min on days 1 and 2 , respectively, and $\sim 90 \mathrm{~min}$ on day 3 . After arrival on day 1, participants confirmed understanding of the procedure and gave written informed consent, filled out questionnaires on demographic variables, and were tested for red-green color blindness using five Ishihara plates (selected from Ishihara, 1990). Before the start of each experimental phase, they were instructed to watch the stimulus presentation with the goal of observing any possible regularity in the occurrence of lamplight colors and electrical stimulation. They were informed that should they discover such a relationship, it would remain stable in all experimental phases (cf, Hermann et al, 2016). This instruction was used to facilitate learning of contingencies (a prerequisite for studying extinction memory recall) and to avoid participants expecting a complete reversal of contingencies during extinction training (ie, expecting stimulation to occur after CS - presentations). However, note that participants were not informed about the actual CS-UCS contingencies.

During all experimental phases, the trial structure was identical for all CS types (except for trials with UCS presentation). After an initial presentation of a black screen with a white fixation cross (duration jittered between 0 and $1.875 \mathrm{~s}$ ), the context without a CS (turned-off lamp) was presented for a duration of $3 \mathrm{~s}$. The CS (lamp within the context picture lighting up in either red, blue, or yellow for the three CS types respectively) was presented for $6 \mathrm{~s}$. During reinforced CS+ trials, the UCS $(100 \mathrm{~ms})$ was delivered immediately after the offset of the CS. A white fixation cross on a black background was shown from CS offset until the start of the next context presentation for 9.125-11 s (total trial duration: $20 \mathrm{~s}$ ).

Acquisition training took place in context A on day 1, extinction training in context $\mathrm{B}$ on day 2 , and recall was tested in contexts $\mathrm{B}$ and $\mathrm{C}$ on day 3. During acquisition training (total duration: $\sim 9 \mathrm{~min}$ ), two separate $\mathrm{CS}+(\mathrm{CS}+\mathrm{E}$ and $\mathrm{CS}+\mathrm{U}$ (see below); eg, red and yellow lights) were shown eight times each, and both CS+ were paired with the UCS in five out of eight trials (62.5\% partial reinforcement rate). A third CS (CS - ; eg, blue light) was never paired with the UCS and shown eight times.

During extinction training (total duration: $\sim 12 \mathrm{~min}$ ), the $\mathrm{CS}+\mathrm{E}$ (extinguished) was shown 16 times without subsequent UCS presentation to extinguish the conditioned fear response. Sixteen CS - trials were presented intermixed with the $16 \mathrm{CS}+\mathrm{E}$ trials, the $\mathrm{CS}+\mathrm{U}$ (unextinguished) was not shown. During recall (total duration: $\sim 17 \mathrm{~min}$ ), all three CS were presented eight times each in context $\mathrm{B}$ as well as in a 
new context $\mathrm{C}$ (within-subjects design) in two blocks with four trials of each CS-context combination, respectively. The first six CS trials always consisted of all three CS in both contexts with a permuted allocation of the first CS. After recall, a reinstatement test was included that is not central to the present research question (see Supplementary Information for further details and Supplementary Figure S1 for a scheme of the paradigm).

Pseudo-randomized stimulus orders were used for all phases, in which no more than two consecutive presentations of the same CS and no more than three consecutive presentations of a CS+ were allowed. During acquisition training, the first and the last three trials consisted of one $\mathrm{CS}-$, one reinforced $\mathrm{CS}+\mathrm{E}$ and one reinforced $\mathrm{CS}+\mathrm{U}$ trial, respectively, whereas the other trials were arranged in three blocks with two trials of each CS (one of the CS+ was not reinforced in each block). During extinction training, the first and last two trials consisted of one CS+E and one CS trial, respectively. In addition, up to three consecutive presentations of the same context were allowed during recall. Individual stimulus presentation orders were used for each participant of the cortisol group and the identical (matched) orders for the placebo group.

\section{Cortisol Administration, Saliva Sampling, and Analysis}

In a double-blind, randomized design, 20 men were administered three $10 \mathrm{mg}$ tablets of cortisol (hydrocortisone; Jenapharm, Germany) $50 \mathrm{~min}$ before the start of the functional scans for extinction on day 2. Visually identical placebos (tablettose and magnesium) were given to the remaining 20 men. In order to assess cortisol concentrations, saliva samples (Salivette sampling devices; Sarstedt, Nümbrecht, Germany) were collected directly before tablet intake (baseline), as well as 40 and $70 \mathrm{~min}$ after tablet intake ( $\sim 5$ min before and immediately after extinction training). In addition, saliva samples were taken at baseline and after the scanning sessions on day 1 ( $\sim 25$ min before and immediately after acquisition training) and day $3(\sim 20 \mathrm{~min}$ before recall and immediately after the reinstatement test; cf, Supplementary Figure S1 in the Supplementary Information). Saliva samples were stored at $-20^{\circ} \mathrm{C}$ until assayed using a Dissociation-Enhanced Lanthanide Fluorescent Immunoassay as described previously (Dressendörfer et al, 1992) determining the fraction of free unbound salivary cortisol. The detection limit was $0.5 \mathrm{nmol} / \mathrm{l}$, and inter- and intra-assay coefficients of variance were below $9.0 \%$ and $6.7 \%$, respectively.

\section{Analyses of Skin Conductance Responses}

Details concerning sampling of skin conductance responses (SCRs) can be found in the Supplementary Information. Statistical comparisons of mean SCRs were conducted separately for acquisition training, extinction training, and recall via analysis of variance (ANOVA). We focused on conditioned responding ( $\mathrm{CS}+\mathrm{E} v s \mathrm{CS}-$ ) to emphasize learning-related contextual responding to extinguished stimuli and added the corresponding analyses regarding the $\mathrm{CS}+\mathrm{U}$ to analyze stimulus generalization. For acquisition and extinction training, the within-subjects factors CS $(\mathrm{CS}+\mathrm{E} / \mathrm{CS}+\mathrm{U}$ vs $\mathrm{CS}-$ ) and block (each comprising four trials of each CS) as well as the between-subjects factor treatment (placebo $v s$ cortisol) were entered. For recall, the first block was analyzed separately for contexts B and C with the factors CS and treatment. We focused on this early recall phase to avoid reextinction processes most likely occurring afterward.

Statistical analyses were performed in IBM SPSS Statistics for Windows 20.0 (Armonk, NY) with Greenhouse-Geisser correction if needed. The statistical significance level was set to $p<0.05$ (trends are reported up to $p<0.077$ ), and effect sizes are reported as $\eta_{p}^{2}$.

\section{fMRI Analyses}

Details concerning data acquisition of brain images, preprocessing, first-level model, and regions of interests (ROIs) can be found in the Supplementary Information. On the second level, random effects group analyses were conducted in Statistical Parametric Mapping (SPM8; Wellcome Department of Cognitive Neurology, London, UK) implemented in MatLab R2012a (Mathworks, Sherborn, MA) using two-sample $t$-tests. Furthermore, the flexible factorial design was used for analyses of time-dependent changes during extinction training.

Functional coupling of the extinction network was analyzed by conducting psychophysiological interaction (PPI) analyses for each subject for early (first block) extinction and early extinction recall. These analyses explore the functional connectivity between a seed region (volume of interest; $5 \mathrm{~mm}$ sphere around the corresponding peak voxel derived from results of the between-group comparisons) and other brain areas in interaction with the experimental task ( $\mathrm{CS}+\mathrm{E} / \mathrm{CS}+\mathrm{U}$ vs $\mathrm{CS}-$ ).

Regarding exploratory whole-brain analyses, the significance threshold was set to $p<0.05$ on voxel-level corrected for multiple testing (family-wise error (FWE) correction); the minimal cluster size $(k)$ was 20 voxels. For the ROI analyses, the significance threshold was set to $p<0.05$ on voxel level, corrected for multiple testing within each ROI (FWEcorrected; using the small volume correction option of SPM8). Results for neural activation (but not functional connectivity) are reported up to $p_{\text {corr. }}<0.10$.

\section{RESULTS}

\section{Participants' Descriptive Data}

No significant differences regarding age or BMI were found between participants in the placebo (mean age \pm SD: $24.8 \pm 4.2$ years; BMI: $24.1 \pm 2.1 \mathrm{~kg} / \mathrm{m}^{2}$ ) compared with the cortisol group (age: $24.1 \pm 2.9$ years; BMI: $23.9 \pm 2.3 \mathrm{~kg} / \mathrm{m}^{2}$; both $p>0.51)$.

\section{Cortisol Concentrations}

Cortisol concentrations changed because of administration of $30 \mathrm{mg}$ hydrocortisone after the baseline sample on day 2 (time $\times$ treatment interaction: $\mathrm{F}_{(1.1,43.6)}=16.24, p<0.001$, $\eta_{p}^{2}=0.299$; main effect time: $\mathrm{F}_{(1.1,43.6)}=15.92, p<0.001$, $\eta_{p}^{2}=0.295$; main effect treatment: $F_{(1,38)}=49.16, p<0.001$, $\eta_{p}^{2}=0.564 ;$ Table 1). Although no differences were evident in the baseline sample $\left(T_{(38)}=0.38, p=0.71\right)$, cortisol concentrations were significantly elevated before $\left(T_{(19.0)}=4.79\right.$, 
$\left.p<0.001, \eta_{p}^{2}=0.376\right)$ and after extinction $\left(T_{(19.0)}=8.91\right.$, $\left.p<0.001, \eta_{p}^{2}=0.676\right)$ in the cortisol compared with the placebo group. Neither on day 1 (fear acquisition) nor on day 3 (recall) any differences between groups or between sampling times were observed (all main and interaction effects: $\mathrm{F}<3.96 ; p>0.05)$.

\section{Fear Acquisition in Context A}

Successful fear acquisition was indicated by a significant differentiation between both $\mathrm{CS}+(\mathrm{CS}+\mathrm{E} / \mathrm{CS}+\mathrm{U})$ and the

Table I Mean (SD) Cortisol Concentrations (in nmol/l) Are Depicted for All 3 Experimental Days

\begin{tabular}{|c|c|c|c|c|c|}
\hline & & \multicolumn{2}{|c|}{ Cortisol } & \multicolumn{2}{|c|}{ Placebo } \\
\hline \multicolumn{2}{|c|}{ Cortisol concentrations } & $\mathbf{M}$ & SD & $\mathbf{M}$ & SD \\
\hline \multirow[t]{2}{*}{ Day I } & Baseline & 4.31 & 2.67 & 5.12 & 4.43 \\
\hline & After acquisition & 3.53 & 3.26 & 4.92 & 4.61 \\
\hline \multirow[t]{3}{*}{ Day 2} & Baseline & 4.38 & 3.53 & 4.01 & 2.62 \\
\hline & Before extinction & |94.8| & 178.96 & 3.11 & 1.50 \\
\hline & After extinction & 113.48 & 55.50 & 2.91 & 1.81 \\
\hline \multirow[t]{2}{*}{ Day 3} & Baseline & 3.98 & 2.10 & 4.64 & 4.40 \\
\hline & After recall & 3.57 & 2.81 & 3.05 & 2.36 \\
\hline
\end{tabular}

On days 1 and 3, no significant differences were found. On day 2, after a comparable baseline, pronounced increases of cortisol concentrations were observed before and after extinction after administration of $30 \mathrm{mg}$ hydrocortisone compared with placebo.
CS -, developing over time $(C S+E$ vs $C S-$ : interaction $\mathrm{CS} \times$ block: $\mathrm{F}_{(1,36)}=24.40, p<0.001, \eta_{p}^{2}=0.404$; main effect CS: $\mathrm{F}_{(1,36)}=26.69, p<0.001, \eta_{p}^{2}=0.426 ; C S+U$ vs $C S-$ : interaction $\mathrm{CS} \times$ block: $\mathrm{F}_{(1,36)}=9.56, p=0.004, \eta_{p}^{2}=0.210$; main effect CS: $\mathrm{F}_{(1,36)}=29.17, p<0.001, \eta_{p}^{2}=0.448$; main effect block: $\mathrm{F}_{(1,36)}=5.12, p=0.030, \eta_{p}^{2}=0.124$; Figure 1). On the neural level, enhanced differential conditioned activation was observed in ACC, right anterior parahippocampal gyrus, left insula, left precentral gyrus, and at a trend level in the left amygdala for the combined CS+ compared with the CS - (Supplementary Table S1 in the Supplementary Information). For both measures, no significant main or interaction effects with treatment were observed.

\section{Extinction Training in Context B}

Differential conditioned responding declined during extinction training (interaction CS $\times$ block: $F_{(2.6,97.9)}=11.76$, $p<0.001, \quad \eta_{p}^{2}=0.236$; main effect CS: $\mathrm{F}_{(1,38)}=15.75$, $p<0.001, \eta_{p}^{2}=0.293$; main effect block: $\mathrm{F}_{(1.6,60.8)}=27.03$, $\left.p<0.001, \quad \eta_{p}^{2}=0.416\right)$. Importantly, cortisol timedependently diminished conditioned responding (interaction CS $\times$ block $\times$ treatment: $\quad \mathrm{F}_{(2.6,97.9)}=3.10, \quad p=0.037$, $\left.\eta_{p}^{2}=0.075\right)$ that was evident in less differential SCRs in the first and second blocks of extinction training in the cortisol compared with the placebo group (both $p<0.05$; Figure 1), but not in the third and fourth blocks (both $p>0.14$ ).

Accordingly, this cortisol effect was also found on the neural level: cortisol significantly altered the time course of extinction learning in the bilateral amygdala, the right

DAY 2

DAY 3

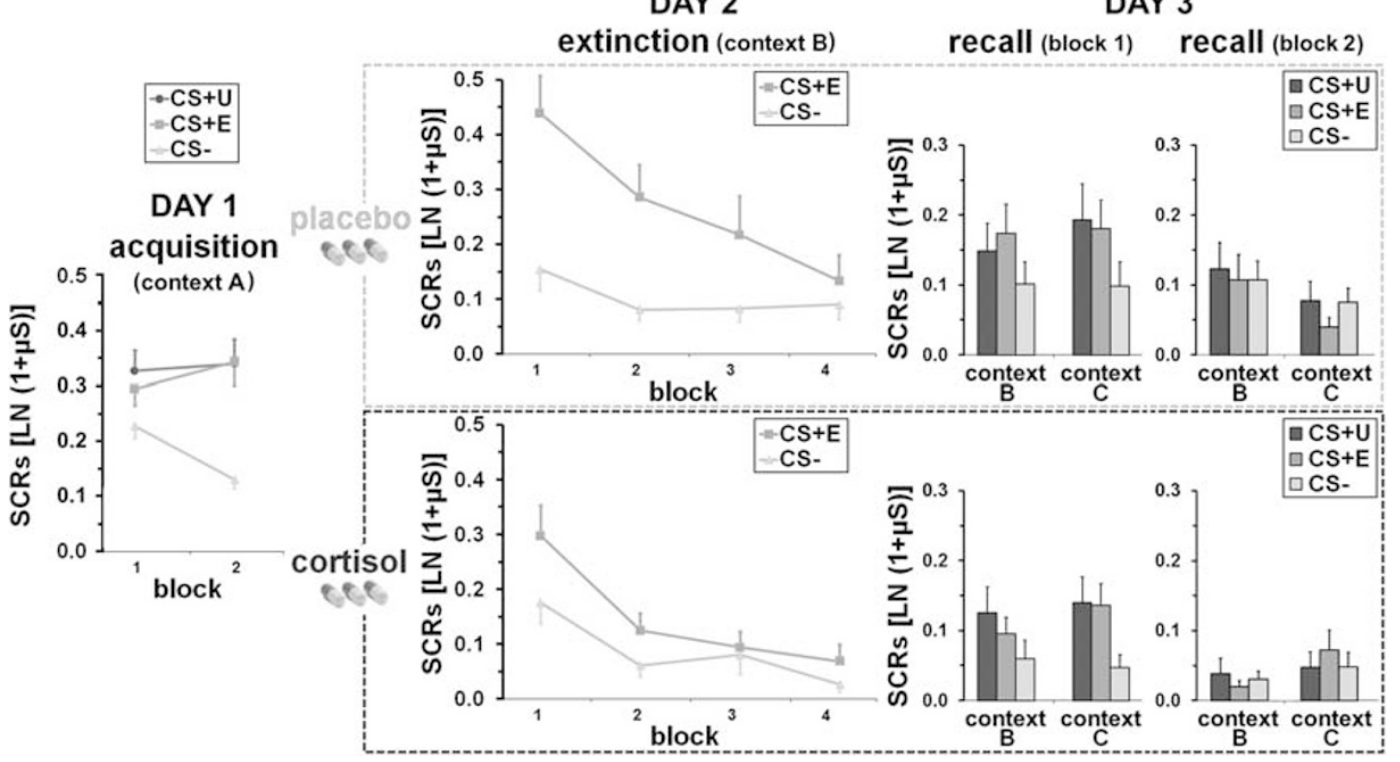

Figure I Mean $( \pm \mathrm{SEM})$ skin conductance responses $(\mathrm{SCRs})$ are depicted for $\mathrm{CS}+\mathrm{E}(\mathrm{CS}+$ extinguished), $\mathrm{CS}+\mathrm{U}(\mathrm{CS}+$ unextinguished), and $\mathrm{CS}-\mathrm{during}$ acquisition in context $A$ on day 1 , extinction in context B on day 2 , and recall in contexts B and $C$ that were presented in an intermixed manner on day 3 after one week. Separate charts are outlined for the placebo (upper row) and cortisol groups (lower row); cortisol administration took place before extinction training. Corresponding to the statistical analyses, all blocks comprise four CS trials. For recall, the first (early) and second (late) blocks are depicted separately for contexts B and C. After successful fear acquisition, conditioned responding was reduced at the beginning of extinction training (first and second blocks) in the cortisol group $(p<0.05)$. For recall in context B, the cortisol group exerted generally reduced SCRs over the first and second blocks compared with the placebo group. When SCRs toward the CS+E and CS - were analyzed between early recall in context B and late extinction training (last block of four trials), the cortisol group also exerted overall reduced SCRs compared with the placebo group. SCRs during recall in context C were not subject to a modulation by cortisol. 
Table 2 Activation Differences for the Contrast CS+E (CS+ Extinguished) Minus CS - Are Displayed for the Time-Dependent Cortisol Effects on Extinction (A) and Group Differences for the First Block of Extinction Training (B)

\begin{tabular}{|c|c|c|c|c|c|}
\hline Structure & $x$ & y & $z$ & $Z_{\max }$ & Pcorr \\
\hline \multicolumn{6}{|l|}{ (A) Interaction treatment $\times$ block } \\
\hline$L$ amygdala & -32 & 0 & -22 & 4.29 & 0.002 \\
\hline R amygdala & 22 & -4 & -28 & 3.40 & 0.042 \\
\hline $\mathrm{R}$ anterior parahippocampal gyrus & 26 & -4 & -32 & 4.17 & 0.005 \\
\hline L insula & -32 & 2 & 14 & 3.53 & 0.094 \\
\hline R hippocampus & 24 & -4 & -28 & 3.75 & 0.027 \\
\hline \multicolumn{6}{|l|}{ (B) First block of extinction training } \\
\hline \multicolumn{6}{|l|}{ Placebo minus cortisol } \\
\hline L amygdala & -24 & -2 & -22 & 2.97 & 0.092 \\
\hline $\mathrm{R}$ amygdala & 22 & -4 & -28 & 3.86 & 0.008 \\
\hline $\mathrm{R}$ anterior parahippocampal & 30 & 0 & -32 & 3.86 & 0.014 \\
\hline \multicolumn{6}{|l|}{ gyrus } \\
\hline R hippocampus & 24 & -4 & -28 & 3.78 & 0.020 \\
\hline \multicolumn{6}{|l|}{ Cortisol minus placebo } \\
\hline No significant results & & & & & \\
\hline
\end{tabular}

$\mathrm{L}=$ left, $\mathrm{R}=$ right.

The significance threshold was set to $p<0.05$ (FWE-corrected for small volume correction). Trends up to $p_{\text {corr }}<0.10$ are reported in italics. All coordinates $(x, y$, z) are given in $\mathrm{MNI}$ space.

anterior parahippocampal gyrus, right hippocampus, and at trend level in the left insula. The post hoc analyses revealed reduced activations in the bilateral amygdala, right anterior parahippocampal gyrus, and right hippocampus during the first block of extinction training in the cortisol compared with the placebo group (Table 2 and Figure 2a). In addition, cortisol heightened the functional connectivity of the right anterior parahippocampal gyrus with the vmPFC during the first block of extinction training compared with placebo $\left(x=-2, y=44, z=-2, Z_{\max }=3.40, p_{\text {corr. }}=0.009\right.$; Figure $\left.2 \mathrm{~b}\right)$. The post hoc analyses revealed that the cortisol group displayed increased functional connectivity between the right anterior parahippocampal gyrus and the $\operatorname{vmPFC}(x=2$, $y=38, z=2, Z_{\max }=3.32, p_{\text {corr. }}=0.012$ ). An additional trend was found in the placebo group showing decreased functional connectivity between the right anterior parahippocampal gyrus and the $\operatorname{vmPFC}(x=4, y=42, z=-4$, $Z_{\text {max }}=2.69, p_{\text {corr. }}=0.068$ ).

\section{Recall in Context B}

Conditioned SCRs significantly recovered for the CS+E (main effect CS: $\mathrm{F}_{(1,36)}=4.94, p=0.033, \eta_{p}^{2}=0.121$ ) as well as for the CS+U (main effect CS: $\mathrm{F}_{(1,36)}=4.70, p=0.037$, $\left.\eta_{p}^{2}=0.115\right)$ in comparison with the CS - . However, no group differences occurred for SCRs in context B (all $p>0.12$; Figure 1). But extending these analyses regarding early recall to the whole recall phase, the cortisol group exhibited reduced overall SCRs compared with the placebo group $\left(C S+E\right.$ vs $C S-$ : main effect treatment: $\mathrm{F}_{(1,36)}=6.07$, $p=0.019, \eta_{p}^{2}=0.144 ; C S+U$ vs $C S-:$ main effect treatment: $\mathrm{F}_{(1,36)}=3.33, \quad p=0.076, \quad \eta_{p}^{2}=0.085 ; \quad$ main effect CS:
$\left.\mathrm{F}_{(1,36)}=5.05, p=0.037, \eta^{2}=0.123\right)$. An additional comparison between conditioned SCRs during early recall in context $\mathrm{B}$ and during late extinction training (fourth block) revealed higher SCRs toward the CS+E compared with the CS (main effect CS: $\mathrm{F}_{(1,36)}=5.80, p=0.021, \eta_{p}^{2}=0.139$ ). Besides, a trend toward overall lowered SCRs in the cortisol group compared with the placebo group was found (main effect treatment: $\left.\mathrm{F}_{(1,36)}=3.48, p=0.070, \eta_{p}^{2}=0.088\right)$. No further main or interaction effects emerged, in particular no $\mathrm{CS} \times$ block interaction. The missing interaction indicates successful recall of the extinction memory, as there was no increase in conditioned SCRs from late extinction training to early recall in context $\mathrm{B}$.

In addition, the cortisol group showed increased differentiation between $\mathrm{CS}+\mathrm{E}$ and $\mathrm{CS}-$ in the right hippocampus $\left(x=22, \quad y=-26, \quad z=-10, \quad Z_{\max }=3.55, \quad p_{\text {corr. }}=0.042\right.$; Figure 3a) and enhanced functional connectivity of this right hippocampal activation peak with the vmPFC $(x=-4$, $y=38, z=0, Z_{\max }=2.95, p_{\text {corr. }}=0.028$; Figure $3 b$ ) during early recall. The post hoc analyses revealed that this result was driven by the cortisol group that exerted increased functional connectivity between the right hippocampus and the vmPFC $\left(x=-4, y=38, z=0, Z_{\max }=3.02, p_{\text {corr. }}=0.026\right)$. For the contrast $\mathrm{CS}+\mathrm{U}$ minus $\mathrm{CS}-$, cortisol reduced neural activation in the right insula $\left(x=36, y=20, z=-8, Z_{\max }=3.88\right.$, $p_{\text {corr. }}=0.026$; Figure $3 c$ ) during early recall. Extending these analyses to the whole recall phase, the reported structures were no longer modulated by cortisol administration.

\section{Recall in Context $\mathrm{C}$}

Conditioned SCRs significantly recovered for the CS+E (main effect CS: $\mathrm{F}_{(1,36)}=15.66, p<0.001, \eta_{p}^{2}=0.303$ ) as well as for the CS+U (main effect CS: $\mathrm{F}_{(1,36)}=15.80, p<0.001$, $\left.\eta_{p}^{2}=0.305\right)$ compared with the CS - during early recall. An additional comparison between conditioned SCRs (CS+E vs CS-) during early recall in context $\mathrm{C}$ and during late extinction training (fourth block) revealed that conditioned SCRs (CS+E vs CS - ) tended to be higher during early recall in context $\mathrm{C}$ compared with late extinction training in context $\mathrm{B}$ (interaction $\mathrm{CS} \times$ block: $\mathrm{F}_{(1,36)}=3.48, p=0.070$, $\eta_{p}^{2}=0.088 ;$ main effect CS: $\mathrm{F}_{(1,36)}=10.14, \quad p=0.003$, $\eta^{2}=0.220 ;$ main effect block: $\mathrm{F}_{(1,36)}=4.14, p=0.049$, $\eta_{p}^{2}=0.103$ ), indicating fear renewal. No further main or interaction effects emerged; importantly, no group differences occurred for SCRs and on the neural level in context C (all $p>0.24$; Figure 1).

Whole-brain analyses did not result in any significant effect for all contrasts.

\section{DISCUSSION}

Cortisol administration before extinction training diminished conditioned fear responses during extinction learning (evident in SCRs and on the neural level). In addition, cortisol altered neural correlates of conditioned responding during recall one week later, also extending to a nonextinguished fear stimulus. However, this cortisol effect did not generalize to a new, unknown context. Overall, these results for the first time provide new insights into how stress hormones influence extinction processes in the long term and demonstrate corresponding boundary conditions. 


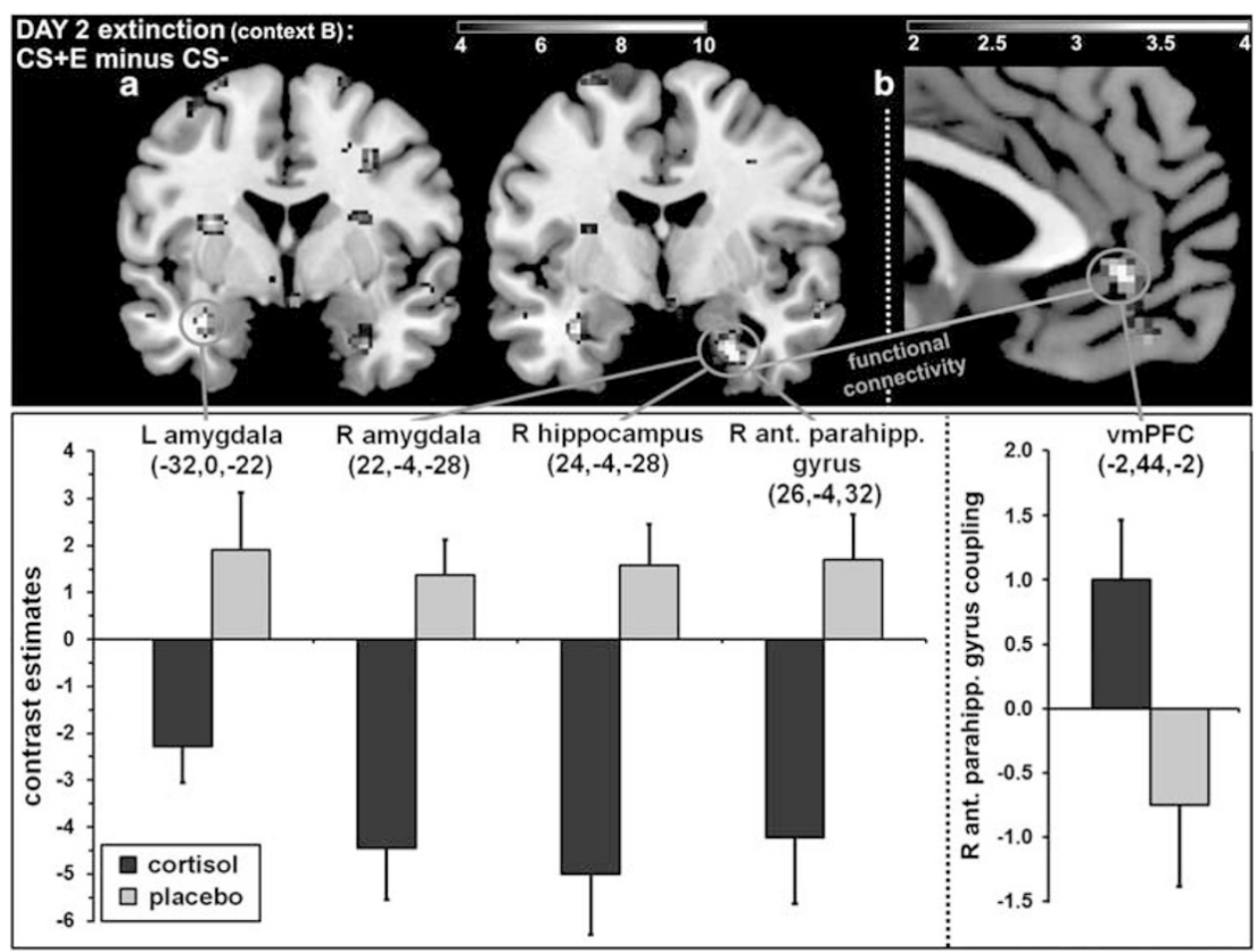

Figure 2 Neural activation and functional connectivity at the beginning of extinction for the contrast CS+E (CS+ extinguished) minus CS - . Data are illustrated with (a) $F \geqslant 4.0$ or (b) $T \geqslant 2.0$ (see color bar for exact $F$ - and $T$-values). The depicted slices were selected according to the reported activations in the left amygdala $(y=0)$, the right amygdala/hippocampus/anterior parahippocampal gyrus $(y=-4)$, and the ventromedial prefrontal cortex (vmPFC; $x=-2)$. Mean $( \pm$ SEM) contrast estimates are displayed in the respective peak voxels in the bar graphs separately for the placebo and the cortisol groups. $L=l$ left, $\mathrm{R}=$ right. (a) Cortisol administration reduced differential neural activation at the beginning of extinction in the amygdala, hippocampus, and anterior parahippocampal gyrus. (b) Furthermore, cortisol enhanced the functional connectivity between the anterior parahippocampal gyrus and the vmPFC.

In line with literature on stress hormone effects on declarative memory (Schwabe et al, 2012) and on exposure therapy (de Quervain et al, 2011; Soravia et al, 2006), we could show that cortisol reduced conditioned SCRs and neural activation in the amygdala-hippocampal complex and enhanced the related functional connectivity to the vmPFC during early extinction learning pointing to inhibited fear recall after cortisol administration. These interactions between amygdala, hippocampus, anterior parahippocampal gyrus, and vmPFC critically reflect the balance between processes conveying fear and extinction memories. All these structures express receptors at which cortisol binds (Joëls and Baram, 2009), hence a modulation of (emotional) learning and memory processes is clearly conceivable (Schwabe et al, 2012). For example, in the rodent model, systemic or intraamygdala stimulation or blockade of cortisol receptors led to facilitated or impaired fear extinction (Ninomiya et al, 2010; Yang et al, 2006, 2007). In men, cortisol administration or stress induction also reduced neural activation in the amygdala-hippocampal complex during fear acquisition and reward anticipation (Kinner et al, 2016; Merz et al, 2012, 2013). Seemingly, cortisol exerts comparable effects on negative as well as on positive emotional processing opening the adjunct treatment application of cortisol to new avenues: not only patients with phobias (de Quervain et al, 2011; Soravia et al, 2006) or PTSD (Aerni et al, 2004; Schelling et al, 2004; Yehuda et al, 2015) might profit from cortisol administration or high cortisol concentrations due to the circadian rhythm (Lass-Hennemann and
Michael, 2014), but also patients with disorders related to aberrant reward processing such as addiction as recently shown in heroin addicts (Walter et al, 2015).

At one week after extinction, we found extinction recall in the extinction context to be changed after cortisol administration as seen in attenuated overall SCRs as well as increases in differential conditioned hippocampal activation and its functional connectivity to the vmPFC. These findings might result from altered consolidation processes taken place after corrected information was acquired during extinction training (cf, Bentz et al, 2013). A concerted action of the hippocampus and vmPFC during extinction recall might be associated with activation of the extinction memory trace and the suppression of the original fear memory trace. This is in line with the proposed role of the hippocampus in conveying contextual information and that of the vmPFC in inhibiting conditioned fear responses via projections to inhibitory intercalated cells in the amygdala (Dejean et al, 2015; Maren et al, 2013; Milad and Quirk, 2012; Quirk and Mueller, 2008). Furthermore, patients with anxiety disorders or PTSD show decreased activation and volumes of the hippocampus and vmPFC (Etkin and Wager, 2007; Graham and Milad, 2011; Greco and Liberzon, 2016) pointing to their essential roles in adequately modulating fear responses.

Furthermore, cortisol reduced insula activation to the $\mathrm{CS}+\mathrm{U}$ during recall in context $\mathrm{B}$. The insula is connected to the amygdala and the ACC (Augustine, 1996), involved in the identification of emotionally important information (Phillips et al, 2003) and the conscious anticipation of 


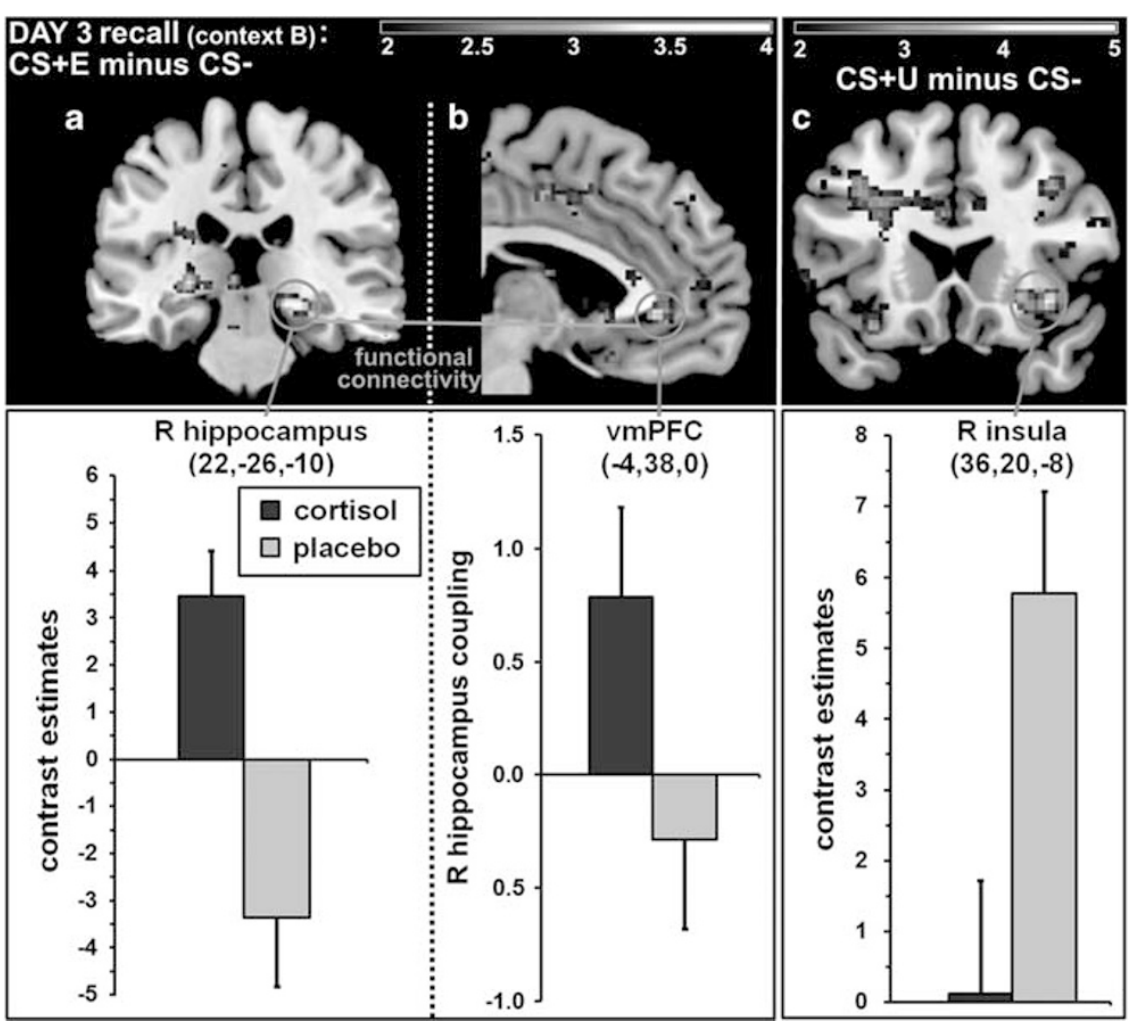

Figure 3 Neural activation and functional connectivity at the beginning of recall in context B for the contrast CS+E (CS+ extinguished) minus CS - as well as neural activation for the contrast CS+U (CS+ unextinguished) minus CS - . Data are illustrated with $T \geqslant 2.0$ (see color bar for exact $T$-values). The depicted slices were selected according to the reported activations in the right hippocampus $(y=-26)$, the ventromedial prefrontal cortex (vmPFC; $x=-4)$, and the right insula $(y=20)$. Mean $( \pm$ SEM) contrast estimates are displayed in the respective peak voxels in the bar graphs separately for the placebo and the cortisol groups. $L=$ left, $R=$ right. (a) Cortisol administration enhanced $C S+E / C S$ - differentiation activation at the beginning of recall in context $B$ in the hippocampus and (b) its functional connectivity to the vmPFC. (c) In the contrast CS+U minus CS - , cortisol reduced neural activation in the right insula.

threat-related stimuli (Mechias et al, 2010; Straube et al, 2007). Importantly, the $C S+U$ was not presented during extinction training, and thus cortisol could not exert a direct effect on $\mathrm{CS}+\mathrm{U}$ processing. It might be assumed that cortisol effects on the recall of extinguished $v s$ unextinguished conditioned responses rely partly on distinct mechanisms.

Cortisol administration before extinction training solely influenced neural activation during early recall, whereas SCRs were generally (irrespective of CS type) reduced only when the whole recall phase was taken into account or when SCRs during early recall were compared with late extinction training (for CS+E vs CS-). These additional analyses at least give a first hint for reduced overall arousal in the cortisol group during recall. Regarding the whole recall phase, it should be noted that reextinction processes might come into play; hence, the observed SCR results cannot be solely attributed to a pure recall effect. Regarding the potentially beneficial effect of cortisol on reextinction, future studies need to test the enduring effects by adding an additional recall phase after reextinction has taken place. Altogether, the interpretation of the recall results should be treated with caution and clearly needs further exploration.

Preextinction cortisol application did not release the extinction memory from its context dependency, as cortisol did not modulate conditioned responding in the new context. Indeed, cortisol diminished SCRs and activation of the amygdala-hippocampal complex during extinction learning and changed extinction recall probably by altering consolidation of the extinction memory. However, these cortisol effects were restricted to the extinction context. For fear extinction as well as extinction of predictions in a neutral learning task, postextinction stress (thus exclusively influencing consolidation) enhanced the context dependency of extinction memories (Hamacher-Dang et al, 2013, 2015) corresponding to our present results. Hence, the exact timing (pre- vs post-extinction stress), memory domain (declarative memory $v s$ fear conditioning), and experimental manipulation (psychosocial stress vs cortisol administration) are crucial factors to consider in future studies. Altogether, our findings complement and extend the proposed model of how exposure therapy benefits from prior cortisol administration (de Quervain and Margraf, 2008; de Quervain et al, 2017) with the corresponding neural underpinnings as well as with stimulus-specific and context-dependent processes.

A necessary prerequisite for cortisol effects on extinction learning to occur seems to include a consolidation phase between acquisition and extinction, as previous work reported cortisol administration directly after acquisition and before extinction to impair extinction learning (Merz et al, 2014). In light of the consolidation hypothesis (McGaugh, 2000), the critical time window of when and how exactly cortisol modulates new (emotional) memories still needs to be determined. Accordingly, time-dependent cortisol effects targeting genomic $v s$ nongenomic pathways 
in the modulation of the amygdala-hippocampus-cortical network should be considered as future research avenues (cf, Henckens et al, 2010, 2011, 2012). Furthermore, the timing between extinction and recall test was set to one week in this experiment to more adequately model long-term processes occurring during exposure therapy. This timing might also have led to a stronger renewal in context $\mathrm{C}$ resulting in enhanced conditioned SCRs toward the $\mathrm{CS}+\mathrm{E}$, comparable to SCRs towards the $\mathrm{CS}+\mathrm{U}$ (cf, Figure 1). Future studies should elucidate whether the same results occur with a different timing of the recall test relative to extinction training also in order to capture long-lasting effects of cortisol on emotional memory. In addition, extinction training took place one day after acquisition training, thus representing delayed extinction that has been associated with attenuated conditioned responding during subsequent recall compared with immediate extinction, in particular in designs with a contextual component (eg, Merz et al, 2016; for a review: Maren, 2014). Hence, we cannot exclude potential floor effects interfering with the possibility to detect betweengroup differences in SCRs in the current design. Besides, SCR analyses were conducted using the same strategy as fMRI analyses, combining the first four trials during recall in one block. Although this strategy is necessary for adequate fMRI analyses, it might not capture more transient effects occurring in SCRs, partly being identifiable only during the first recall trial (cf, Lonsdorf et al, 2017). Altogether, neural and behavioral findings during recall in this study did not correspond completely, complicating a clear interpretation of the neural findings on day 3 .

In conclusion, cortisol administration before extinction training influences subsequent extinction learning and context-dependent fear and extinction recall: on one side, cortisol decreased conditioned fear at the beginning of extinction training; on the other side, cortisol seems to influence extinction memory consolidation resulting in a neural activation pattern one week later, most likely reflecting stronger inhibitory processes, but without generalizing to the behavioral level. Neural underpinnings of these effects comprise key players of the fear and extinction network and their interplay: amygdala, hippocampal complex, and vmPFC. We provide first evidence that cortisol appears to also influence a nonextinguished stimulus but only within the extinction context. Thus, contextualization of extinction memories still comprises a challenge to be faced in order to further enhance treatment success of CBT.

\section{FUNDING AND DISCLOSURE}

This work was supported by a grant from the German Research Foundation (DFG; ME 3831/4-1 to CJM and HE 7013/1-1 to AH). The DFG had no further role in study design, collection, analysis and interpretation of data, in the writing of the manuscript, and in the decision to submit the paper for publication. The authors declare no conflict of interest.

\section{ACKNOWLEDGMENTS}

We thank Andreas Haltermann, Jessica Heinen, Eve-Mariek Hessas, Alina Renner, and Adam Schweda for help in participant recruitment and data collection. We acknowledge the support of the Neuroimaging Centre of the Research Department of Neuroscience at the Ruhr-University Bochum and PHILIPS Germany. In addition, we thank the members of the biochemical laboratory of the Department of Biological and Clinical Psychology (University of Trier) for saliva analyses and Sabine Kagerer for language editing.

\section{REFERENCES}

Aerni A, Traber R, Hock C, Roozendaal B, Schelling G, Papassotiropoulos A et al (2004). Low-dose cortisol for symptoms of posttraumatic stress disorder. Am J Psychiatry 161: 1488-1490.

Augustine JR (1996). Circuitry and functional aspects of the insular lobe in primates including humans. Brain Res Rev 22: 229-244.

Bentz D, Michael T, Wilhelm FH, Hartmann FR, Kunz S, von Rohr IRR et al (2013). Influence of stress on fear memory processes in an aversive differential conditioning paradigm in humans. Psychoneuroendocrinology 38: 1186-1197.

Boschen MJ, Neumann DL, Waters AM (2009). Relapse of successfully treated anxiety and fear: theoretical issues and recommendations for clinical practice. Aust N Z J Psychiatry 43: 89-100.

Bouton ME (2002). Context, ambiguity, and unlearning: sources of relapse after behavioral extinction. Biol Psychiatry 52: 976-986.

Bouton ME (2004). Context and behavioral processes in extinction. Learn Mem 11: 485-494.

Craske MG, Kircanski K, Zelikowsky M, Mystkowski J, Chowdhury $\mathrm{N}$, Baker A (2008). Optimizing inhibitory learning during exposure therapy. Behav Res Ther 46: 5-27.

de Quervain DJ-F, Bentz D, Michael T, Bolt OC, Wiederhold BK, Margraf J et al (2011). Glucocorticoids enhance extinction-based psychotherapy. Proc Natl Acad Sci USA 108: 6621-6625.

de Quervain DJ-F, Margraf J (2008). Glucocorticoids for the treatment of post-traumatic stress disorder and phobias: a novel therapeutic approach. Eur J Pharmacol 583: 365-371.

de Quervain DJ-F, Schwabe L, Roozendaal B (2017). Stress, glucocorticoids and memory: implications for treating fearrelated disorders. Nat Rev Neurosci 18: 7-19.

Dejean C, Courtin J, Rozeske RR, Bonnet MC, Dousset V, Michelet $\mathrm{T}$ et al (2015). Neuronal circuits for fear expression and recovery: recent advances and potential therapeutic strategies. Biol Psychiatry 78: 298-306.

Dressendörfer RA, Kirschbaum C, Rohde W, Stahl F, Strasburger CJ (1992). Synthesis of a cortisol-biotin conjugate and evaluation as a tracer in an immunoassay for salivary cortisol measurement. J Steroid Biochem Mol Biol 43: 683-692.

Etkin A, Wager TD (2007). Functional neuroimaging of anxiety: a meta-analysis of emotional processing in PTSD, social anxiety disorder, and specific phobia. Am J Psychiatry 164: 1476-1488.

Graham BM, Milad MR (2011). The study of fear extinction: implications for anxiety disorders. Am J Psychiatry 168: $1255-1265$.

Greco JA, Liberzon I (2016). Neuroimaging of fear-associated learning. Neuropsychopharmacology 41: 320-334.

Hamacher-Dang TC, Merz CJ, Wolf OT (2015). Stress following extinction learning leads to a context-dependent return of fear. Psychophysiology 52: 489-498.

Hamacher-Dang TC, Uengoer M, Wolf OT (2013). Stress impairs retrieval of extinguished and unextinguished associations in a predictive learning task. Neurobiol Learn Mem 1-8.

Henckens MJAG, van Wingen GA, Joëls M, Fernandez G (2010). Time-dependent effects of corticosteroids on human amygdala processing. J Neurosci 30: 12725-12732.

Henckens MJAG, van Wingen GA, Joëls M, Fernandez G (2011). Time-dependent corticosteroid modulation of prefrontal 
working memory processing. Proc Natl Acad Sci USA 108: 5801-5806.

Henckens MJAG, van Wingen GA, Joëls M, Fernandez G (2012). Corticosteroid induced decoupling of the amygdala in men. Cereb Cortex 22: 2336-2345.

Hermann A, Stark R, Milad MR, Merz CJ (2016). Renewal of conditioned fear in a novel context is associated with hippocampal activation and connectivity. Soc Cogn Affect Neurosci 11: 1411-1421.

Ishihara S (1990). Ishihara's tests for color-blindness, 38 plate ed. Kanehara, Shuppan: Tokyo/Kyoto.

Joëls M, Baram TZ (2009). The neuro-symphony of stress. Nat Rev Neurosci 10: 459-466.

Kessler RC, Berglund P, Demler O, Jin R, Merikangas KR, Walters EE (2005). Lifetime prevalence and age-of-onset distributions of DSM-IV disorders in the national comorbidity survey replication. Arch Gen Psychiat 62: 593-602.

Kinner VL, Wolf OT, Merz CJ (2016). Cortisol alters reward processing in the human brain. Horm Behav 84: 75-83.

Lass-Hennemann J, Michael T (2014). Endogenous cortisol levels influence exposure therapy in spider phobia. Behav Res Ther 60: $39-45$.

Lonsdorf TB, Menz M, Andreatta M, Fullana M, Golkar A, Haaker J et al (2017). Don't fear 'fear conditioning': methodological considerations for the design and analysis of studies on human fear acquisition, extinction, and return of fear. Neurosci Biobehav Rev 77: 247-285.

Maren S (2014). Nature and causes of the immediate extinction deficit: a brief review. Neurobiol Learn Mem 113: 19-24.

Maren S, Phan KL, Liberzon I (2013). The contextual brain: implications for fear conditioning, extinction and psychopathology. Nat Rev Neurosci 14: 417-428.

McGaugh JL (2000). Memory - a century of consolidation. Science 287: 248-251.

Mechias M-L, Etkin A, Kalisch R (2010). A meta-analysis of instructed fear studies: implications for conscious appraisal of threat. Neuroimage 49: 1760-1768.

Merz CJ, Hamacher-Dang TC, Wolf OT (2016). Immediate extinction promotes the return of fear. Neurobiol Learn Mem 131: 109-116.

Merz CJ, Hermann A, Stark R, Wolf OT (2014). Cortisol modifies extinction learning of recently acquired fear in men. Soc Cogn Affect Neurosci 9: 1426-1434.

Merz CJ, Tabbert K, Schweckendiek J, Klucken T, Vaitl D, Stark R et al (2012). Oral contraceptive usage alters the effects of cortisol on implicit fear learning. Horm Behav 62: 531-538.

Merz CJ, Wolf OT, Schweckendiek J, Klucken T, Vaitl D, Stark R (2013). Stress differentially affects fear conditioning in men and women. Psychoneuroendocrinology 11: 2529-2541.

Milad MR, Quirk GJ (2012). Fear extinction as a model for translational neuroscience: ten years of progress. Annu Rev Psychol 63: 129-151.
Milad MR, Wright CI, Orr SP, Pitman RK, Quirk GJ, Rauch SL (2007). Recall of fear extinction in humans activates the ventromedial prefrontal cortex and hippocampus in concert. Biol Psychiatry 62: 446-454.

Nakataki M, Soravia LM, Schwab S, Horn H, Dierks T, Strik W et al (2016). Glucocorticoid administration improves aberrant fear processing networks in spider phobia. Neuropsychopharmacology 42: 485-494.

Ninomiya EM, Martynhak BJ, Zanoveli JM, Correia D, da Cunha C, Andreatini R (2010). Spironolactone and low-dose dexamethasone enhance extinction of contextual fear conditioning. Prog Neuropsychopharmacol Biol Psychiatry 34: $1229-1235$.

Oldfield RC (1971). The assessment and analysis of handedness: The Edinburgh inventory. Neuropsychologia 9: 97-113.

Phillips ML, Drevets WC, Rauch SL, Lane R (2003). Neurobiology of emotion perception I: The neural basis of normal emotion perception. Biol Psychiatry 54: 504-514.

Quirk GJ, Mueller D (2008). Neural mechanisms of extinction learning and retrieval. Neuropharmacology 33: 56-72.

Schelling G, Kilger E, Roozendaal B, de Quervain DJ-F, Briegel J, Dagge A et al (2004). Stress doses of hydrocortisone, traumatic memories, and symptoms of posttraumatic stress disorder in patients after cardiac surgery: a randomized study. Biol Psychiatry 55: 627-633.

Schwabe L, Joëls M, Roozendaal B, Wolf OT, Oitzl MS (2012). Stress effects on memory: an update and integration. Neurosci Biobehav Rev 36: 1740-1749.

Soravia LM, Heinrichs M, Aerni A, Maroni C, Schelling G, Ehlert U et al (2006). Glucocorticoids reduce phobic fear in humans. Proc Natl Acad Sci USA 103: 5585-5590.

Straube T, Mentzel H-J, Miltner WHR (2007). Waiting for spiders: Brain activation during anticipatory anxiety in spider phobics. Neuroimage 37: 1427-1436.

Vervliet B, Craske MG, Hermans D (2013). Fear extinction and relapse: state of the art. Annu Rev Clin Psychol 9: 215-248.

Walter M, Bentz D, Schicktanz N, Milnik A, Aerni A, Gerhards C et al (2015). Effects of cortisol administration on craving in heroin addicts. Transl Psychiatry 5: e610.

Yang Y-L, Chao P-K, Lu K-T (2006). Systemic and intra-amygdala administration of glucocorticoid agonist and antagonist modulate extinction of conditioned fear. Neuropsychopharmacology 31: 912-924.

Yang Y-L, Chao P-K, Ro L-S, Wo Y-YP, Lu K-T (2007). Glutamate NMDA receptors within the amygdala participate in the modulatory effect of glucocorticoids on extinction of conditioned fear in rats. Neuropsychopharmacology 32: 1042-1051.

Yehuda R, Bierer LM, Pratchett LC, Lehrner A, Koch EC, van Manen JA et al (2015). Cortisol augmentation of a psychological treatment for warfighters with posttraumatic stress disorder: randomized trial showing improved treatment retention and outcome. Psychoneuroendocrinology 51: 589-597.

Supplementary Information accompanies the paper on the Neuropsychopharmacology website (http://www.nature.com/npp) 